\title{
Intra-Dialytic Hemoglobin Changes and Cardiovascular Events: A Cohort Study on Dialysis Outcomes and Practice Patterns in Japan
}

\author{
Takashi Hara $^{\text {a, b }}$ Miho Kimachi ${ }^{a}$ Tatsuyoshi Ikenoue ${ }^{c}$ Tadao Akizawa $^{d}$ \\ Shunichi Fukuhara ${ }^{\mathrm{a}}$ e Yosuke Yamamoto $^{\mathrm{a}}$ \\ a Department of Healthcare Epidemiology, Graduate School of Medicine and Public Health, Kyoto University,

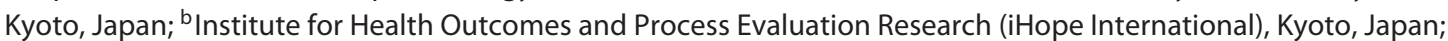 \\ 'Department of Human Health Science, Graduate School of Medicine, Kyoto University, Kyoto, Japan; ${ }^{\mathrm{d} D i v i s i o n}$ of \\ Nephrology, Showa University School of Medicine, Tokyo, Japan; ${ }^{e}$ Center for Innovative Research for Communities \\ and Clinical Excellence (CIRC2LE), Fukushima Medical University, Fukushima, Japan
}

\section{Keywords}

Hemodialysis · Ultrafiltration · Plasma refilling ·

Hemoglobin · Cardiovascular events

\begin{abstract}
Background: Ultrafiltration during hemodialysis (HD) causes hemoconcentration. Little is known about the relationships between intra-dialytic changes in hemoglobin concentration and cardiovascular events. Thus, this study aimed to elucidate the relationships between intra-dialytic changes in hemoglobin concentration and cardiovascular events among HD patients. Methods: This prospective cohort study was based on the Japanese Dialysis Outcomes and Practice Pattern Study phases 4 and 5. The predictor was the ratio of post-dialysis hemoglobin concentration to pre-dialysis hemoglobin concentration (post-Hb/pre-Hb) at baseline. The primary outcome was major adverse cardiovascular events (MACEs). Hazard ratios (HRs) were estimated using a Cox model for the association between post-Hb/pre-Hb and MACEs, adjusting for potential confounders. Results: A total of 865 patients were enrolled. During a median follow-up of 2.6 years, 145 (16.8\%) patients developed MACEs. Patients were divided into 4 categories according to baseline post-
\end{abstract}

\begin{tabular}{ll}
\hline KARGER & (c) 2019 The Author(s) \\
Published by S. Karger AG, Basel Operger \\
E-Mail karger@karger.com & This article is licensed under the Creative Commons Attribution- \\
www.karger.com/ajn & $\begin{array}{l}\text { NonCommercial-NoDerivatives 4.0 International License (CC BY- } \\
\text { NC-ND) (http://www.karger.com/Services/OpenAccessLicense). } \\
\text { Usage and distribution for commercial purposes as well as any dis- } \\
\text { tribution of modified material requires written permission. }\end{array}$
\end{tabular}

$\mathrm{Hb} /$ pre-Hb $(<1.0, \geq 1.0$ to $<1.1, \geq 1.1$ to $<1.2$, and $\geq 1.2)$. The multivariable-adjusted HRs for MACEs were $1.69(95 \% \mathrm{Cl}$ 1.36-2.10), 1.29 (95\% Cl 1.10-1.51), and 1.31 (95\% Cl 1.021.68 ) in patients with post-Hb/pre-Hb ratios of $<1.0, \geq 1.0$ to $<1.1$, and $\geq 1.2$, respectively, compared with the reference post- $\mathrm{Hb}$ /pre-Hb ratio of $\geq 1.1$ to $<1.2$. Cubic spline analyses revealed a U-shaped association between post-Hb/pre-Hb and MACEs. Conclusion: High and low intra-dialytic changes in hemoglobin concentration are associated with a high risk of MACEs in patients undergoing HD.

C 2019 The Author(s)
Published by S. Karger AG, Basel

\section{Introduction}

The aim of hemodialysis (HD) is to remove waste, uremic toxins, and fluid from the blood by diffusion and ultrafiltration. Fluid removal is usually performed by ultrafiltration and the aim is to achieve a clinical "dry weight (DW)" [1]. Fluid removal for patients undergoing $\mathrm{HD}$ is one of the major challenges. Previous studies on fluid removal in HD patients have pointed to an association between an interdialytic weight loss 
(IDWL) $>5.7 \%$ of post-dialytic body weight and higher mortality [2]. Furthermore, a high ultrafiltration rate $(>10 \mathrm{~mL} / \mathrm{kg} / \mathrm{h})$ is associated with high mortality $[3,4]$. In fact, a recent statement from the Chief Medical Officers of US dialysis providers suggests that appropriate fluid management is one of the most important unmet issues for contemporary HD patients globally; moreover, this statement promotes "volume first," meaning that volume control should be the primary goal of dialysis care [5].

Fluid removal also results in hemoconcentration and increases hemoglobin concentration after HD [6]. The previous observational study showed that each $1 \mathrm{~L}$ of ultrafiltration led to an increase in hemoglobin concentration of approximately $0.4 \mathrm{~g} / \mathrm{dL}$. However, the correlation between IDWL and the hemoglobin increment was moderate (correlation coefficient: $r=0.527$ ) [6]. Some patients show significant increased hemoglobin concentration and others show none at all after fluid removal. In the situation of increased hemoglobin concentration, the ultrafiltration volume exceeds the refill volume from the interstitial spaces into the vascular space. However, when the ultrafiltration volume exceeds the refill volume significantly, this results in intravascular volume depletion and causes potentially intradialytic morbid events, such as muscle cramping and intradialytic hypotension $[7,8]$. These events are known risk factors for cardiovascular events [9]. On the other hand, no change in hemoglobin concentration during dialysis as measured with a continuous monitor such as CRIT-LINE, is interpreted as volume overload in accordance with the previous studies $[10,11]$. A status of fluid overload is also a well-known risk factor for mortality and cardiovascular events $[12,13]$. Intra-dialytic changes in hemoglobin concentration may reflect changes in intravascular fluid volume [14] and may provide an index for balance of ultrafiltration and fluid refilling when it assesses the ratio between post-dialysis hemoglobin concentration and pre-dialysis hemoglobin concentration (post$\mathrm{Hb} /$ pre-Hb). Our hypothesis was that some increase in this index might be good practice but too much of an increase or no change at all may still carry high risk of cardiovascular events.

To our knowledge, there is no study about the relationship between intra-dialytic changes in hemoglobin concentration - defined as the ratio between post-Hb/pre$\mathrm{Hb}$ concentration and cardiovascular events.

Hence, to clarify this relationship, we conducted a cohort study using data from the Dialysis Outcomes and Practice Pattern Study (DOPPS) in Japan.

Intra-Dialytic Hemoglobin Changes and Cardiovascular Events

\section{Materials and Methods}

\section{Study Design and Population}

DOPPS was a prospective cohort study of patients enrolled randomly from a representative sample of dialysis facilities within each participating country. All participants in DOPPS provided written informed consent prior to study enrollment. Detailed information on the design of DOPPS has been provided previously $[15,16]$. Our cohort study used the Japanese DOPPS (J-DOPPS), which was approved by a central Ethics Committee. The current study design was approved by Kyoto University Graduate School and Faculty of Medicine Kyoto University Hospital Ethics Committee (approval number R1301). Data for the current analysis were obtained from J-DOPPS4 (2009-2011) and J-DOPPS5 (2012-2014). The study included patients undergoing maintenance $\mathrm{HD}$ who were older than 18 years and had available data on post-Hb/pre-Hb concentrations. We excluded patients with hemoglobin concentrations $<7.0 \mathrm{~g} / \mathrm{dL}$ because there was some possibility of the requirement for blood transfusion clinically.

\section{Predictor}

The predictor was the ratio of post-Hb/pre-Hb concentration, which was calculated as follows:

\section{Post-Hb concentration/Pre-Hb concentration}

(hereinafter called "post-Hb/pre-Hb”)

$=$ post $-\mathrm{Hb}$ volume $/$ (pre dialysis intravascular volume - ultrafiltration volume + refill volume)/pre-Hb volume/pre dialysis intravascular volume

$=$ pre dialysis intravascular volume/pre dialysis intravascular volume - ultrafiltration volume + refill volume

$=$ pre dialysis intravascular volume/post dialysis intravascular volume

This index shows the intra-dialytic change in intravascular fluid volume in a simple fashion. When pre-Hb/post-Hb volumes are nearly equal, they cancel each other out. For example, when the pre-Hb concentration is $10.0 \mathrm{~g} / \mathrm{dL}$ and the post-Hb concentration is $11.0 \mathrm{~g} / \mathrm{dL}$, the index is 1.1 . This means that intra-dialytic intravascular fluid loss led to a 1.1-fold hemoconcentration. Hemoglobin concentrations were assessed in the first session of the week at the beginning of the observation period. Patients were divided into 4 categories by 0.1 increments as follows: $(1)<1.0,(2) \geq 1.0$ to $<1.1$, (3) $\geq 1.1$ to $<1.2$, and $(4) \geq 1.2$. The 0.1 increment was based on a $2.5 \mathrm{~kg}$ IDWL patient with a pre-Hb concentration of $10.7 \mathrm{~g} / \mathrm{dL}$. The $2.5 \mathrm{~kg}$ IDWL level was derived from the recommended interdialytic weight gain and the pre-Hb concentration of $10.7 \mathrm{~g} / \mathrm{dL}$ was derived from the mean pre-Hb level based on exhaustive data for HD patients in Japan $[17,18]$. According to a previous study [6], each $1 \mathrm{~L}$ of ultrafiltration led to an increase in hemoglobin concentration of approximately $0.4 \mathrm{~g} / \mathrm{dL}$. Therefore, based on this, the pre- $\mathrm{Hb}$ concentration was estimated to increase by $1.0 \mathrm{~g} / \mathrm{dL}$, whereas the post-Hb/pre-Hb was estimated to increase by approximately $0.1 \mathrm{~g} / \mathrm{dL}$. A post-Hb/pre-Hb $=1.0$ meant that the ultrafiltration volume equaled the refill volume. A post-Hb/pre-Hb $>1.0$ 
meant that the ultrafiltration volume exceeded the refill volume. A post-Hb/pre- $\mathrm{Hb}=1.1$ was used as a reference in our model for continuous variable analysis and a post- $\mathrm{Hb} /$ pre- $\mathrm{Hb}$ ratio of $\geq 1.1$ to $<1.2$ was used as a reference in our model for categorical variable analysis.

\section{Outcomes}

The primary outcome was major adverse cardiovascular events (MACEs), including acute myocardial infarction, stroke, and all-cause mortality [19]. We have included all-cause death as a composite outcome because substance causes of death among HD patients were related to cardiovascular events [20, 21]. The secondary outcome was all-cause mortality and vascular access failure.

\section{Statistical Analysis}

With respect to the baseline characteristics of patients categorized by post-Hb/pre-Hb ratio, continuous data with a normal distribution were summarized as means SD; continuous variables with skewed data, as medians (interquartile range); and dichotomous data, as proportions. The distribution of baseline data according to the categorized post-Hb/pre- $\mathrm{Hb}$ ratio was compared using trend analysis. The event-free survival rate for MACEs, allcause mortality, and vascular access failure according to the categorized post-Hb/pre-Hb ratio was described by the Kaplan-Meier method and compared using the log-rank test. Unadjusted and multivariable-adjusted hazard ratios (HRs) with 95\% CI for MACEs, all-cause mortality, and vascular access failure according to the post-Hb/pre-Hb ratio were calculated using a Cox proportional hazards model. The assumption of proportional hazards was graphically checked using a log cumulative hazard plots for each outcome according to the post- $\mathrm{Hb} / \mathrm{pre}-\mathrm{Hb}$ ratio. The multivariable-adjusted model was adjusted for age, sex, cause of end-stage renal disease (ESRD), history of heart disease, HD vintage, IDWL, treatment time, ultrafiltration rate, serum albumin, serum phosphorus, pre-Hb concentration, pre-dialysis systolic blood pressure (BP), changes in systolic BP before and after dialysis, and postdialysis body weight. These variables were based on a priori clinical judgment and existing studies. We used robust variance estimates to consider cluster effects at the facility level. Furthermore, we analyzed the relationship nonlinearly by modeling the post-Hb/pre$\mathrm{Hb}$ ratio as a continuous variable rather than a categorical value using a restricted cubic spline. We used 4 knots located at the 5 th, $35 \mathrm{th}, 65 \mathrm{th}$, and 95 th percentiles of the post-Hb/pre-Hb ratio, as recommended [22].

\section{Sensitivity Analysis}

A multiple imputation approach was used to account for missing covariates. Twenty imputations were performed using the multiple imputations with chained equations methods, assuming that analyzed data were missing at random [23]. These estimates were combined using Rubin's rules. We analyzed imputed missing covariate data in the same way as for primary outcome (Sensitivity analysis 1). In addition, we analyzed imputed missing covariate data, including C-reactive protein (CRP) data, in the same way as for the primary outcome (Sensitivity analysis 2). Furthermore, we defined the predictor as mean baseline data and data after 4 months, and we conducted the same analysis as for the primary outcome (Sensitivity analysis 3). We performed a survival analysis after 4 months from study initiation.
All analyses were performed using Stata software version 15.0 (Stata Corp., College Station, TX, USA). $p$ values $<0.05$ were considered to indicate statistical significance.

\section{Results}

\section{Baseline Characteristics}

A total of 865 patients were enrolled in the study (online suppl. Fig. 1; for all online suppl. material, see www. karger.com/doi/10.1159/000502633). The mean age was 65.6 years, $61.4 \%$ were male, $36.3 \%$ had ESRD caused by diabetes, and the median duration of dialysis therapy was 3.6 years (Table 1). Patients with higher post-Hb/ pre-Hb ratios showed longer dialysis vintage, longer treatment time, higher serum phosphorus, a higher proportion of IDWL $>5.7 \%$ of post-dialytic body weight, a higher proportion of ultrafiltration rate $>10 \mathrm{~mL} / \mathrm{kg} / \mathrm{h}$, higher pre-dialysis systolic BP, and greater changes in systolic BP before and after dialysis. In contrast, patients with lower post-Hb/pre-Hb ratios were older, with a higher proportion of ESRD caused by diabetes. The missing data for each variable are shown in online supplementary Table 1 .

\section{Association between Post-Hb/Pre-Hb and Risk of \\ MACEs, All-Cause Mortality, and Vascular Access \\ Failure}

Categorical Variable Analysis

During a median follow-up of 2.6 years, 145 (16.8\%) patients developed MACEs. The details of MACEs are shown in online supplementary Table 2. Survival rates according to post-Hb/pre-Hb ratio are shown in Figure 1a. The Kaplan-Meier survival estimates of patients from varying post-Hb/pre-Hb categories differed among all 4 groups $(p<0.001)$. The unadjusted HRs for MACEs were 1.73 (95\% CI 1.40-2.14), 1.33 (95\% CI 1.10-1.61), and 1.17 (95\% CI 0.95-1.43) in patients with post-Hb/pre-Hb ratios of $<1.0, \geq 1.0$ to $<1.1$, and $\geq 1.2$ respectively. Furthermore, after adjustment for potential confounders, adjusted HRs for MACEs were 1.69 (95\% CI 1.36-2.10), 1.29 (95\% CI 1.10-1.51), and 1.31 (95\% CI 1.02-1.68) in patients with post-Hb/pre-Hb ratios of $<1.0, \geq 1.0$ to $<1.1$, and $\geq 1.2$ respectively (Table 2 ).

With regard to mortality, during a median follow-up of 2.6 years, $116(13.4 \%)$ patients died (all-cause). The causes of death are shown in online supplementary Table 2. Event-free survival rates according to post-Hb/ pre-Hb ratio are shown in Figure $1 \mathrm{~b}$. The association between the 4 categories of post-Hb/pre-Hb ratio and 
Table 1. Baseline characteristics according to post-Hb/pre-Hb categories

\begin{tabular}{|c|c|c|c|c|c|c|}
\hline & $\begin{array}{l}\text { Total } \\
(n=865)\end{array}$ & $\begin{array}{l}<1.0 \\
(n=115)\end{array}$ & $\begin{array}{l}\geq 1.0 \text { to }<1.1 \\
(n=348)\end{array}$ & $\begin{array}{l}\geq 1.1 \text { to }<1.2 \\
(n=314)\end{array}$ & $\begin{array}{l}\geq 1.2 \\
(n=88)\end{array}$ & $p$ for trend \\
\hline Age, years & $65.6(12.5)$ & $67.1(12.0)$ & $66.6(12.1)$ & $64.8(12.6)$ & $62.6(14.1)$ & 0.003 \\
\hline Gender, male & 61.4 & 64.4 & 65.5 & 56.4 & 59.1 & 0.055 \\
\hline Hypertension & 86.1 & 82.6 & 85.6 & 86.9 & 89.7 & 0.130 \\
\hline Diabetes & 42.3 & 53.9 & 42.0 & 39.8 & 37.5 & 0.016 \\
\hline History of cerebrovascular disease & 15.1 & 13.9 & 18.1 & 13.7 & 10.2 & 0.186 \\
\hline History of heart disease & 45.9 & 42.6 & 44.3 & 47.1 & 52.3 & 0.128 \\
\hline Cause of ESRD: diabetes & 36.3 & 46.1 & 36.2 & 34.7 & 29.6 & 0.019 \\
\hline Vascular access: arteriovenous fistula & 89.3 & 90.7 & 89.6 & 89.7 & 84.7 & 0.277 \\
\hline Hemodialysis vintage, years & $3.6(0.6-8.3)$ & $1.6(0.2-7.1)$ & $2.7(0.5-7.1)$ & $4.6(1.4-10.5)$ & $5.7(1.3-11.7)$ & $<0.001$ \\
\hline IDWL $>5.7 \%$ of post-dialytic body weight & 9.6 & 1.7 & 8.3 & 10.5 & 21.6 & $<0.001$ \\
\hline Ultrafiltration rate $>\mathrm{I} 0 \mathrm{~mL} / \mathrm{kg} / \mathrm{h}$ & 43.1 & 21.7 & 36.8 & 51.0 & 68.2 & $<0.001$ \\
\hline Treatment time, minutes & $230.7(29.3)$ & $223.6(30.6)$ & $229.6(28.6)$ & $232.0(28.2)$ & $239.9(32.2)$ & 0.001 \\
\hline Pre-dialysis $\mathrm{Hb}, \mathrm{g} / \mathrm{dL}$ & $10.4(1.2)$ & $10.4(1.3)$ & $10.4(1.2)$ & $10.4(1.1)$ & $10.6(1.2)$ & 0.174 \\
\hline Serum albumin, $\mathrm{g} / \mathrm{dL}$ & $3.7(0.5)$ & $3.7(0.5)$ & $3.7(0.5)$ & $3.7(0.3)$ & $3.7(0.4)$ & 0.423 \\
\hline Serum total calcium, mg/dL & $8.8(0.8)$ & $8.8(0.8)$ & $8.9(0.8)$ & $8.8(0.7)$ & $8.9(0.7)$ & 0.965 \\
\hline Serum phosphorus, mg/dL & $5.3(1.3)$ & $5.1(1.3)$ & $5.3(1.3)$ & $5.4(1.2)$ & $5.7(1.5)$ & 0.006 \\
\hline Intact $\mathrm{PTH}, \mathrm{pg} / \mathrm{mL}$ & $133.3(74.1-215.0)$ & $157.0(99.0-227.0)$ & $125.0(72.0-208.0)$ & $135.5(74.1-216.5)$ & $143.0(74.6-194.0)$ & 0.802 \\
\hline $\mathrm{CRP}, \mathrm{mg} / \mathrm{dL}$ & $0.12(0.05-0.41)$ & $0.10(0.05-0.30)$ & $0.11(0.05-0.32)$ & $0.15(0.07-0.49)$ & $0.10(0.05-0.42)$ & 0.592 \\
\hline Pre-dialysis systolic BP, $\mathrm{mm} \mathrm{Hg}$ & $147.4(23.8)$ & $153.4(25.6)$ & $148.2(23.6)$ & $144.8(22.1)$ & $146.0(26.6)$ & 0.010 \\
\hline Changes in systolic $\mathrm{BP}$ before and after dialysis, $\mathrm{mm} \mathrm{Hg}$ & $7.8(23.9)$ & $2.1(22.2)$ & $5.3(23.1)$ & $9.5(23.6)$ & $19.4(25.5)$ & $<0.001$ \\
\hline Post-dialysis body weight, $\mathrm{kg}$ & $54.9(12.0)$ & $54.2(11.0)$ & $55.0(12.4)$ & $54.7(11.4)$ & $56.3(13.6)$ & 0.276 \\
\hline
\end{tabular}

Values for categorical variables are given as percentages. Values for continuous variables are given as mean (SD) or median (interquartile range). Patients were divided into 4 categories $(<1.0, \geq 1.0$ to $<1.1, \geq 1.1$ to $<1.2$, and $\geq 1.2)$.

Post-Hb/pre-Hb, post-dialysis hemoglobin concentration to pre-dialysis hemoglobin concentration; ESRD, end-stage renal disease; IDWL, interdialytic weight loss; Hb, hemoglobin; PTH, parathyroid hormone; CRP, C-reactive protein; BP, blood pressure.

mortality is shown in Table 2. Similarly, compared with the reference, the unadjusted HRs for all-cause mortality were 1.76 (95\% CI 1.44-2.16), 1.32 (95\% CI 1.10 1.59), and 1.15 (95\% CI 0.95-1.39) in patients with post- $\mathrm{Hb} /$ pre-Hb ratios of $<1.0, \geq 1.0$ to $<1.1$, and $\geq 1.2$, respectively, compared with the reference $(\geq 1.1$ to $<1.2)$. Furthermore, adjusted HRs for all-cause mortality were 1.71 (95\% CI 1.37-2.12), 1.29 (95\% CI 1.10 $1.50)$, and 1.28 (95\% CI 1.01-1.62) in patients with post-Hb/pre-Hb ratios of $<1.0, \geq 1.0$ to $<1.1$, and $\geq 1.2$ respectively.

With regard to vascular access failure, during a median follow-up of 2.3 years, 106 (12.3\%) patients developed vascular access failure. Event-free survival rates according to post- $\mathrm{Hb} /$ pre- $\mathrm{Hb}$ ratio are shown in Figure 1c. Similarly, compared with the reference, the unadjusted HRs for vascular access failure were 1.78 (95\% CI 1.442.20), 1.32 (95\% CI 1.09-1.59), and 1.11 (95\% CI $0.93-$ 1.33 ) in patients with post-Hb/pre-Hb ratios of $<1.0, \geq 1.0$ to $<1.1$, and $\geq 1.2$, respectively, compared with the reference $(\geq 1.1$ to $<1.2)$. Furthermore, adjusted HRs for vascular access failure were 1.69 (95\% CI 1.37-2.09), 1.27 (95\% CI $1.07-1.50)$, and 1.20 (95\% CI $0.95-1.52)$ in patients with post-Hb/pre-Hb ratios of $<1.0, \geq 1.0$ to $<1.1$, and $\geq 1.2$ respectively.

Intra-Dialytic Hemoglobin Changes and Cardiovascular Events
Continuous Variable Analysis

Multivariable-adjusted restricted cubic spline analyses revealed a U-shaped association between post-Hb/pre$\mathrm{Hb}$ ratio and MACEs, all-cause mortality and vascular access failure (Fig. 2).

Sensitivity Analysis

In the dataset, imputed missing covariate data, adjusted HRs for MACEs were 1.69 (95\% CI 1.41-2.02), 1.28 (95\% CI 1.08-1.52), and 1.35 (95\% CI 1.07-1.71) in patients with post-Hb/pre-Hb ratios of $<1.0, \geq 1.0$ to $<1.1$, and $\geq 1.2$, respectively, compared with the reference (online suppl. Table 3 ).

In the dataset, imputed missing covariate data, including CRP, compared with the reference, the adjusted HRs for MACEs were 1.69 (95\% CI 1.402.03), 1.27 (95\% CI 1.08-1.51), and 1.33 (95\% CI $1.05-$ $1.68)$ in patients with post-Hb/pre-Hb ratios of $<1.0$, $\geq 1.0$ to $<1.1$, and $\geq 1.2$ respectively (online suppl. Table 3 ).

In the dataset in which the predictor was defined as the mean baseline and data after 4 months, a total of 831 patients were enrolled. During a median followup of 2.6 years, $136(16.4 \%)$ patients developed MACEs. The adjusted HRs for MACEs were 1.77 (95\% CI 1.332.36), 1.27 (95\% CI 1.11-1.46), and 1.38 (95\% CI $1.11-$ 


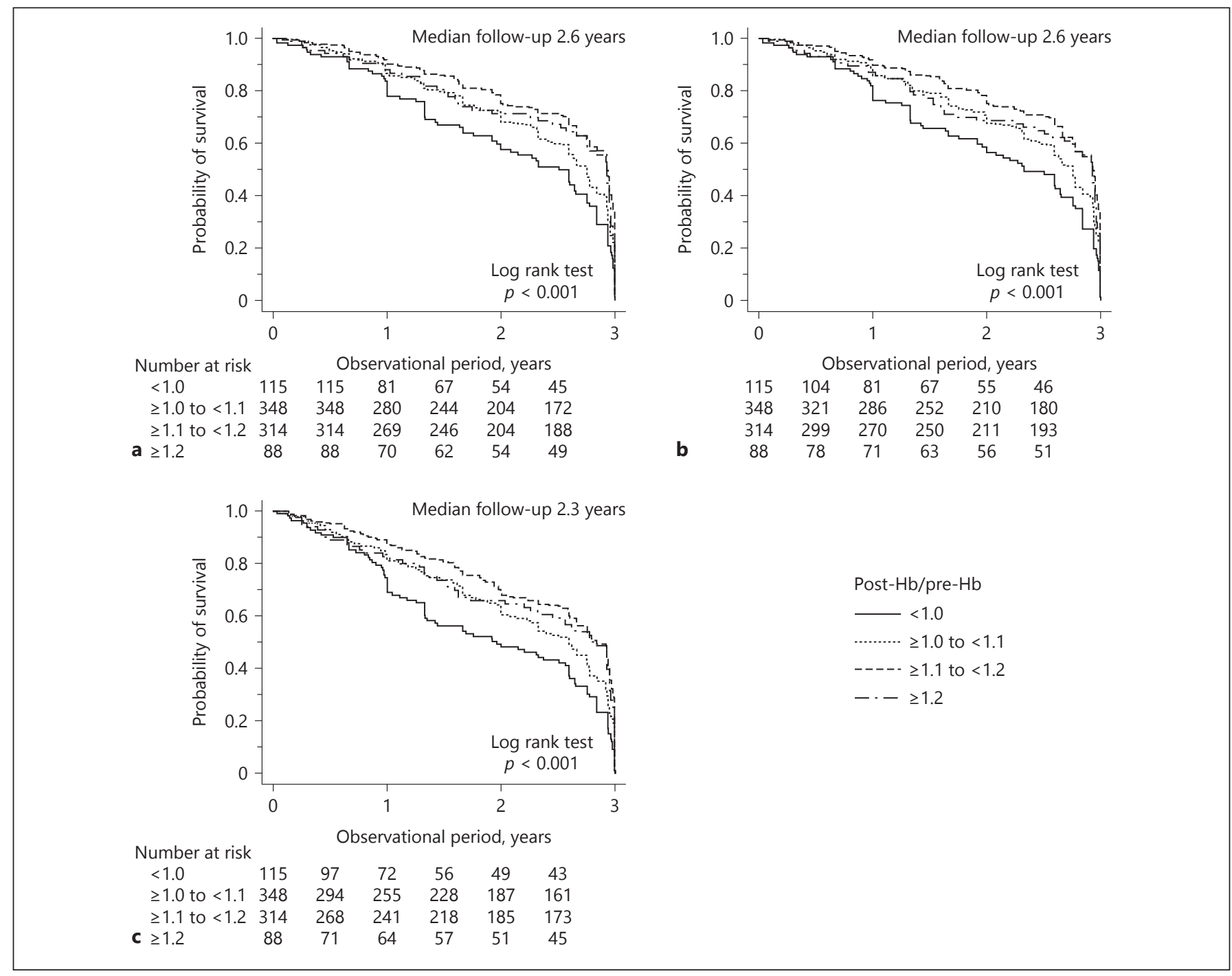

Fig. 1. Event-free survival rate for (a) MACEs, (b) all-cause mortality and (c) vascular access failure by post- $\mathrm{Hb} /$ pre- $\mathrm{Hb}$ categories during the follow-up period. MACEs included acute myocardial infarction, stroke, and all-cause mortality. MACEs, major adverse cardiovascular events; post-Hb/pre-Hb, post-dialysis hemoglobin concentration to pre-dialysis hemoglobin concentration.
1.72 ) in patients with post- $\mathrm{Hb} /$ pre- $\mathrm{Hb}$ ratios of $<1.0$, $\geq 1.0$ to $<1.1$, and $\geq 1.2$ respectively (online suppl. Table 3).

Similar results were obtained for the continuous associations between post-Hb/pre-Hb ratio and MACEs in all datasets (online suppl. Fig. 2).

\section{Discussion}

In this study, we found that both high and low intradialytic changes in hemoglobin concentration were associated with higher risk of MACEs in patients undergoing
HD. The continuous variable analysis revealed a Ushaped association between intra-dialytic changes in hemoglobin concentration and MACEs. Consistent associations were shown in both categorical and continuous analyses. Additionally, similar results regarding this association were also obtained in the sensitivity analysis.

The association between low intra-dialytic changes in hemoglobin concentration and high mortality risk is consistent with that reported in a previous study [24]. On the other hand, the associations between high intradialytic change in hemoglobin concentration and high cardiovascular events and mortality risk are new findings. The mechanisms may be explained as follows. Pre- 
Table 2. HRs for MACEs, all-cause mortality and vascular access failure by post-Hb/pre-Hb categories

\begin{tabular}{|c|c|c|c|c|}
\hline \multicolumn{5}{|l|}{ MACEs } \\
\hline Unadjusted HR & $1.73(1.40-2.14)$ & $1.33(1.10-1.61)$ & Reference & $1.17(0.95-1.43)$ \\
\hline Adjusted HR & $1.69(1.36-2.10)$ & $1.29(1.10-1.51)$ & Reference & $1.31(1.02-1.68)$ \\
\hline \multicolumn{5}{|l|}{ All-cause mortality } \\
\hline Unadjusted HR & $1.76(1.44-2.16)$ & $1.32(1.10-1.59)$ & Reference & $1.15(0.95-1.39)$ \\
\hline Adjusted HR & $1.71(1.37-2.12)$ & $1.29(1.10-1.50)$ & Reference & $1.28(1.01-1.62)$ \\
\hline Unadjusted HR & $1.78(1.44-2.20)$ & $1.32(1.09-1.59)$ & Reference & $1.11(0.93-1.33)$ \\
\hline Adjusted HR & $1.69(1.37-2.09)$ & $1.27(1.07-1.50)$ & Reference & $1.20(0.95-1.52)$ \\
\hline
\end{tabular}

Bold values statistically significant values. The multivariable-adjusted model was adjusted for age, sex, cause of end-stage renal disease, history of heart disease, hemodialysis vintage, IDWL, treatment time, ultrafiltration rate, serum albumin, serum phosphorus, pre$\mathrm{Hb}$ concentration, pre-dialysis systolic BP, changes in systolic BP before and after dialysis, and post-dialysis body weight.

MACEs included acute myocardial infarction, stroke, and all-cause mortality.

$\mathrm{HR}$, hazard ratio; MACEs, major adverse cardiovascular events; post-Hb/pre-Hb, post-dialysis hemoglobin concentration topredialysis hemoglobin concentration; IDWL, interdialytic weight loss; BP, blood pressure.

vious studies have suggested that the change in hemodynamics during HD caused by the effects of ultrafiltration on hemoglobin concentration is a potential cause of intra-dialytic hypotension that results in decreased perfusion of important organs, such as the heart and brain $[25,26]$. Decreased perfusion of vital organs results in damage in each HD session. We presume that the ultrafiltration condition that results in high changes in hemoglobin concentrations during HD may be not appropriate.

In addition, this index may be useful as a tool to decide whether the DW is appropriate. In 1967, DW was initially defined as "the reduction of BP to hypotensive levels during ultrafiltration and unassociated with other obvious causes" by Thomson et al. [27]. Since then, the definition has changed [28]. In 2009, Sinha and Agarwal [29] proposed this definition: "DW is defined as the lowest tolerated post-dialysis weight achieved via gradual change in post-dialysis weight at which there are minimal signs or symptoms of hypovolemia or hypervolemia." Considering the latest DW definition, in some patients with low intra-dialytic changes in hemoglobin concentration, true DW would be lower. In contrast, in some patients with high intra-dialytic changes in hemoglobin concentration, true DW would be higher.

Finally, the association between intra-dialytic changes in hemoglobin concentration and MACEs remained even with adjustments for IDWL and ultrafiltration rate. Considering the index formula described in the Methods section, the association between intra-dialytic changes in hemoglobin concentration and cardiovascular events may indicate the association between plasma refilling and cardiovascular events. Plasma refilling cannot be measured easily in the clinical setting [30]. Of course, this study did not directly measure plasma refilling. However, we presume that there is an association between plasma refilling and cardiovascular events due to the influence of injury to the endothelial glycocalyx, which plays a central role in the fluid movement [31].

The strengths of this study are as follows. First, this research was a prospective study with a relatively large sample size. Second, the sampling approach of J-DOPPS renders it representative of most Japanese dialysis settings. Third, this index is very easy to use clinically. Through this simple method, post-dialysis intravascular fluid balance can be evaluated by calculating the ratio of post-Hb/pre-Hb concentration.

However, there were several limitations to this study. First, patients may have transferred into the different categories because baseline data were used to define exposure categories in this cohort. However, similar results were obtained when we defined the predictor as mean baseline data and data after 4 months and conducted the same analysis as for primary outcome.

Second, measurements were performed during the first dialysis session of the week when patients were at maximum volume overload and maximum hemodilution. The proportion of patients with high intra-dialytic changes in hemoglobin concentration was likely to decrease around the mid-week dialysis session, and this may have affected the results. However, in practice, when clinicians perform fluid removal, they attempt to 

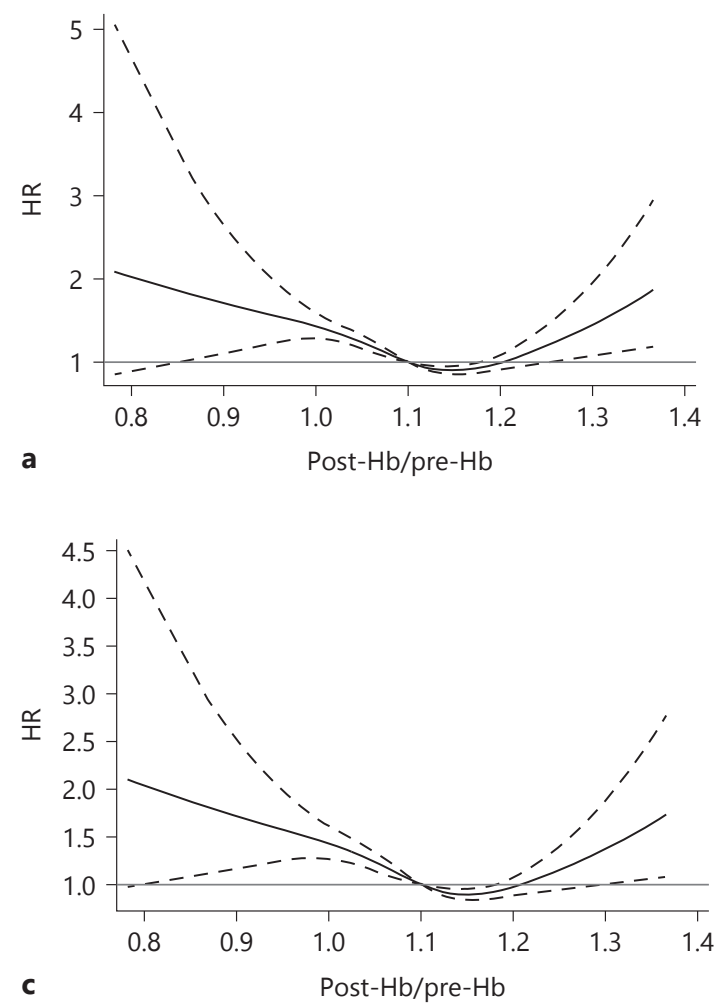

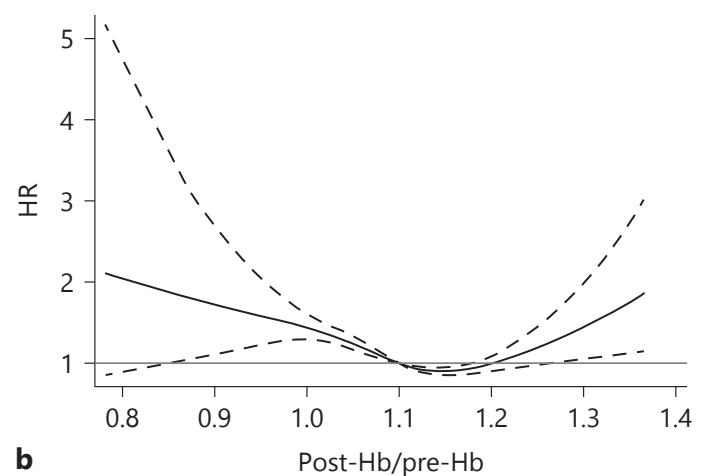

Post-Hb/pre-Hb
Fig. 2. HRs for MACEs, all-cause mortality, and vascular access failure by post-Hb/pre-Hb ratio. Multivariable-adjusted restricted cubic spline plots of the HR for (a) MACEs, (b) all-cause mortality, and (c) vascular access failure according to post- $\mathrm{Hb} /$ pre- $\mathrm{Hb}$ ratio. The solid line represents $\mathrm{HR}$, and the dotted line represents $95 \%$ CI. The horizontal gray line corresponds to the normal reference $\mathrm{HR}$ of 1.0. Post-Hb/pre-Hb ratio $=1.1$ was used as reference in this study. The multivariable-adjusted model was adjusted for

achieve a clinical DW in all dialysis sessions. Further studies considering the timing of measurements are warranted.

Third, we lacked information on 2 major confounding factors, CRP and residual renal function (RRF), in our study. CRP, as an inflammatory factor, could not be adjusted for in our model of main analysis because about $41 \%$ of CRP data was missing from this dataset. However, similar results were obtained using imputed missing CRP data, assuming that analyzed data were missing at random (online suppl. Table 3). RRF would affect post-Hb/ pre-Hb ratios through fluid removal. In practice, clinicians tend to aim for lower IDWL and lower ultrafiltration rates for patients with sufficient RRF, which might age, sex, cause of ESRD, history of heart disease, HD vintage, IDWL, treatment time, ultrafiltration rate, serum albumin, serum phosphorus, pre- $\mathrm{Hb}$, pre-dialysis systolic $\mathrm{BP}$, changes in systolic $\mathrm{BP}$ before and after dialysis, and post-dialysis body weight. MACEs included acute myocardial infarction, stroke, and all-cause mortality. MACEs, major adverse cardiovascular events; post-Hb/pre$\mathrm{Hb}$, post-dialysis hemoglobin concentration to pre-dialysis hemoglobin concentration; HR, hazard ratio.

result in low intra-dialytic changes in hemoglobin concentration. The presence of RRF is associated with a lower mortality risk [32]. Therefore, we might have underestimated the association between low intra-dialytic changes in hemoglobin concentration and higher mortality risk. Fourth, post-Hb was measured in only $20 \%$ of patients in the J-DOPPS 4-5. Therefore, there may be some characteristic differences in the analyzed patients compared with all patients in the J-DOPPS $4-5$, which may have led to selection bias (online suppl. Table 1). Fifth, this study was observational, and therefore, it could not provide direct cause-and-effect associations.

In conclusion, our study revealed a U-shaped association between intra-dialytic changes in hemoglobin con- 
centration and MACEs among patients undergoing maintenance HD. Our results may contribute to future recommendations regarding appropriate fluid removal conditions and appropriate DW.

\section{Acknowledgment}

We appreciate the cooperation of the patients and facilities participating in the J-DOPPS.

\section{Statement of Ethics}

All participants in J-DOPPS provided written informed consent prior to study enrollment. The study design was approved by the appropriate ethics review board.

\section{Disclosure Statement}

T.A. has been a scientific advisor or consultant for Astellas Pharma, JT Pharmaceuticals, Torii Pharmaceutical, Kyowa Hakko Kirin, Nipro Medical, Ono Pharmaceutical, Bayer HealthCare, Fuso Pharmaceutical, GlaxoSmithKline, and Kissei Pharmaceuti- cal and has received lecture fees from Chugai Pharmaceutical, Kyowa Hakko Kirin, Bayer HealthCare, Torii Pharmaceutical, Kissei Pharmaceutical, and Ono Pharmaceutical. T.H., M.K., T.I., S.F., and Y.Y. have no conflicts of interest to declare.

\section{Funding Sources}

The DOPPS is coordinated by Arbor Research Collaborative for Health and J-DOPPS is supported by scientific research grants from Kyowa Hakko Kirin Co., Ltd., without any restrictions on publication. The funder had no role in the study design, data collection and analysis, decision to publish, or preparation of the manuscript.

\section{Author Contributions}

T.H., M.K., T.I., and S.F.: research idea and study design. T.H. and M.K.: data acquisition. T.H., M.K., and Y.Y.: data analysis/interpretation. T.H., M.K., and Y.Y.: statistical analysis. T.A. and S.F.: supervision or mentorship. Each author contributed important intellectual content during manuscript drafting or revision and accepts accountability for the overall work by ensuring that questions pertaining to the accuracy or integrity of any portion of the work are appropriately investigated and resolved.

\section{References}

1 Jaeger JQ, Mehta RL. Assessment of dry weight in hemodialysis: an overview. J Am Soc Nephrol. 1999 Feb;10(2):392-403.

2 Saran R, Bragg-Gresham JL, Rayner HC, Goodkin DA, Keen ML, Van Dijk PC, et al. Nonadherence in hemodialysis: associations with mortality, hospitalization, and practice patterns in the DOPPS. Kidney Int. $2003 \mathrm{Jul}$; 64(1):254-62.

3 Assimon MM, Wenger JB, Wang L, Flythe JE. Ultrafiltration rate and mortality in maintenance hemodialysis patients. Am J Kidney Dis. 2016 Dec;68(6):911-22.

4 Saran R, Bragg-Gresham JL, Levin NW, Twardowski ZJ, Wizemann V, Saito A, et al. Longer treatment time and slower ultrafiltration in hemodialysis: associations with reduced mortality in the DOPPS. Kidney Int. 2006 Apr;69(7):1222-8.

5 Weiner DE, Brunelli SM, Hunt A, Schiller B, Glassock R, Maddux FW, et al. Improving clinical outcomes among hemodialysis patients: a proposal for a "volume first" approach from the chief medical officers of US dialysis providers. Am J Kidney Dis. 2014 Nov;64(5):685-95.

6 Bellizzi V, Minutolo R, Terracciano V, Iodice C, Giannattasio P, De Nicola L, et al. Influence of the cyclic variation of hydration status on hemoglobin levels in hemodialysis patients. Am J Kidney Dis. 2002 Sep;40(3):549-55.
7 Stewart WK, Fleming LW, Manuel MA. Muscle cramps during maintenance haemodialysis. Lancet. 1972 May;1(7759):1049-51.

8 van der Sande FM, Kooman JP, Leunissen KM. Intradialytic hypotension-new concepts on an old problem. Nephrol Dial Transplant. 2000 Nov;15(11):1746-8.

9 MacEwen C, Sutherland S, Daly J, Pugh C, Tarassenko L. Relationship between hypotension and cerebral ischemia during hemodialysis. J Am Soc Nephrol. 2017 Aug;28(8):2511-20.

10 Steuer RR, Leypoldt JK, Cheung AK, Harris $\mathrm{DH}$, Conis JM. Hematocrit as an indicator of blood volume and a predictor of intradialytic morbid events. ASAIO J. 1994 Jul-Sep; 40(3):M691-6.

11 Steuer RR, Leypoldt JK, Cheung AK, Senekjian HO, Conis JM. Reducing symptoms during hemodialysis by continuously monitoring the hematocrit. Am J Kidney Dis. 1996 Apr; 27(4):525-32.

12 Wizemann V, Wabel P, Chamney P, Zaluska W, Moissl U, Rode C, et al. The mortality risk of overhydration in haemodialysis patients. Nephrol Dial Transplant. 2009 May;24(5): 1574-9.

13 Lee MJ, Doh FM, Kim CH, Koo HM, Oh HJ, Park JT, et al. Interdialytic weight gain and cardiovascular outcome in incident hemodialysis patients. Am J Nephrol. 2014;39(5): 427-35.
14 Movilli E, Pertica N, Camerini C, Cancarini GC, Brunori G, Scolari F, et al. Predialysis versus postdialysis hematocrit evaluation during erythropoietin therapy. Am J Kidney Dis. 2002 Apr;39(4):850-3.

15 Young EW, Goodkin DA, Mapes DL, Port FK, Keen ML, Chen K, et al. The Dialysis Outcomes and Practice Patterns Study (DOPPS): an international hemodialysis study. Kidney Int. 2000 Jan;57(suppl 74): S74-81.

16 Pisoni RL, Gillespie BW, Dickinson DM, Chen K, Kutner MH, Wolfe RA. The Dialysis Outcomes and Practice Patterns Study (DOPPS): design, data elements, and methodology. Am J Kidney Dis. 2004 Nov;44(5 suppl 2):7-15.

17 Tischer CC, Wilcox CS. Nephrology, 2nd ed. Baltimore (MD): Williams \& Wilkins; 1993.

18 Masakane I, Taniguchi M, Nakai S, Tsuchida K, Wada A, Ogata S, et al. Annual Dialysis Data Report 2016, JSDT Renal Data Registry. Ren Replace Ther. 2018;4(1):45.

19 Wang C, Kane R, Levenson M, Kelman J, Wernecke M, Lee JY, et al. Association between changes in CMS reimbursement policy and drug labels for erythrocyte-stimulating agents with outcomes for older patients undergoing hemodialysis covered by fee-forservice Medicare. JAMA Intern Med. 2016 Dec;176(12):1818-25. 
20 Go AS, Chertow GM, Fan D, McCulloch CE, Hsu CY. Chronic kidney disease and the risks of death, cardiovascular events, and hospitalization. N Engl J Med. 2004 Sep;351(13): 1296-305.

21 Matsubara Y, Kimachi M, Fukuma S, Onishi Y, Fukuhara S. Development of a new risk model for predicting cardiovascular events among hemodialysis patients: Populationbased hemodialysis patients from the Japan Dialysis Outcome and Practice Patterns Study (J-DOPPS). PLoS One. 2017 Mar; 12(3): e0173468.

22 Harrell FE. General aspects of fitting regression models. Regression modeling strategies: with applications to linear models, logistic regression, and survival analysis, 2nd ed. New York (NY): Springer New York; 2001. pp. 13-44.

23 van Buuren S, Boshuizen HC, Knook DL. Multiple imputation of missing blood pressure covariates in survival analysis. Stat Med. 1999 Mar; 18(6):681-94.
24 Nishiwaki H, Hasegawa T, Koiwa F, Hamano $\mathrm{T}$, Masakane I. The association of the difference in hemoglobin levels before and after hemodialysis with the risk of 1-year mortality in patients undergoing hemodialysis. Results from a nationwide cohort study of the Japanese Renal Data Registry. PLoS One. 2019 Jan; 14(1):e0210533.

25 Stefanidis I, Bach R, Mertens PR, Liakopoulos $\mathrm{V}$, Liapi G, Mann H, et al. Influence of hemodialysis on the mean blood flow velocity in the middle cerebral artery. Clin Nephrol. 2005 Aug;64(2):129-37.

26 McIntyre CW, Burton JO, Selby NM, Leccisotti L, Korsheed S, Baker CS, et al. Hemodialysis-induced cardiac dysfunction is associated with an acute reduction in global and segmental myocardial blood flow. Clin J Am Soc Nephrol. 2008 Jan;3(1):19-26.

27 Thomson GE, Waterhouse K, McDonald HP Jr, Friedman EA. Hemodialysis for chronic renal failure. Clinical observations. Arch Intern Med. 1967 Aug;120(2):153-67.
28 Agarwal R, Weir MR. Dry-weight: a concept revisited in an effort to avoid medication-directed approaches for blood pressure control in hemodialysis patients. Clin J Am Soc Nephrol. 2010 Jul;5(7):1255-60.

29 Sinha AD, Agarwal R. Can chronic volume overload be recognized and prevented in hemodialysis patients? The pitfalls of the clinical examination in assessing volume status. Semin Dial. 2009 Sep-Oct;22(5):480-2.

30 Levick JR, Michel CC. Microvascular fluid exchange and the revised Starling principle. Cardiovasc Res. 2010 Jul;87(2):198-210.

31 Dane MJ, Khairoun M, Lee DH, van den Berg BM, Eskens BJ, Boels MG, et al. Association of kidney function with changes in the endothelial surface layer. Clin J Am Soc Nephrol. 2014 Apr;9(4):698-704.

32 Obi Y, Rhee CM, Mathew AT, Shah G, Streja E, Brunelli SM, et al. Residual kidney function decline and mortality in incident hemodialysis patients. J Am Soc Nephrol. 2016 Dec; 27(12):3758-68. 